\title{
The Role of Tyramine Within the Mouse Ovary
}

\author{
S. Peck ${ }^{1}$, S.M.B. Obayomi ${ }^{1}$ and D.P. Baluch ${ }^{1, *}$ \\ 1. School of Life Sciences, Arizona State University, Tempe, Arizona, USA. \\ * Corresponding author: page.baluch@asu.edu
}

Complications associated with ovulation account for nearly $40 \%$ of infertility cases in women, yet the specific cause of ovarian fecundity has yet to be identified. Understanding the signaling pathways that control the maturation and release of oocytes from the ovary may provide insight into how the ovarian cycle can be further regulated, especially for women experiencing infertility due to ovarian dysfunction. Previous studies have shown that the uterus, which is composed of innervated smooth muscle, can be induced to contract by the biogenic monoamine tyramine at levels similar to estrogen [1]. Tyramine is known to be associated with peripheral vasoconstriction, increased cardiac output, and increased respiration, which are all affected by smooth muscle activity [2]. Within the ovary, ovarian follicles possess numerous cell types that share common morphological features with smooth muscle such as the tunica albuginea, the chordae, and the theca externa. These follicles are surrounded by a ring of smooth muscle like cells, which play a role in the cycling of oocytes within the ovary and ultimately the expulsion from the follicle. Because tyramine can modulate uterine contractions, this project focused on what role tyramine may have in the ovary by measuring the levels of tyramine using HPLC, and observing morphological changes using histology and immunohistochemistry.

Mouse tissue used in this project was approved through the IACUC protocol 18-1606R at Arizona State University and are obtained from a $\mathrm{C} 57 \mathrm{BL} / 6 \mathrm{~J}$ mouse line. Protocols for superovulation, procurement and preparing tissue for immunohistochemistry are detailed in a previous publication [3]. Ovaries were taken from eight-week-old female mice and labeled with an antibody against the Trace Amine-Associated Receptor 1 (TAAR1), which is a specific receptor for tyramine (ThermoFisher, OSR00119W) and with phalloidin (ThermoFisher, A12380), a toxin from the Amanita phalloides mushroom, conjugated to Alexa 568, which selectively binds F-actin. Tyramine levels in the ovary were confirmed by HPLC and found to be at higher concentrations in mice that had not been injected with the hormones PMSG (pregnant mare's serum gonadotropin) and hCG (human chorionic gonadotropin), which are used for superovulation. Scans of the ovary were taken as a set of tile images with a Leica SP5 confocal microscope using a $20 \mathrm{x}$ objective through a depth of $201 \mathrm{~m}$ at $1 / \mathrm{m}$ step sections.

Upon hormonal super-ovulation, immature eggs within the ovary called oocytes are cycled from meiotic metaphase I to meiotic metaphase II and are released into the oviduct. A super-ovulated mouse ovary can release 10-15 oocytes during this process, which results in a deflated appearing ovary (Fig 1C). TAAR1 receptor was generally distributed throughout the ovary prior to super-ovulation (Fig 1A) but upon stimulation, concentrates in the thickened areas called the corpus luteum where eggs have been released (Fig 1B-D). Because the follicles are surrounded by smooth muscle, it was predicted that stimulation using a physiological concentration of tyramine could alter the organization of the staged oocytes or possibly initiate their ejection. In Fig 2B, a tyramine treated ovary contains disrupted actin enriched borders around the follicles and many contain a thickened corpus luteum which results after oocyte ejection similar to super-ovulation. Further studies will focus on how tyramine may be involved in the process of oocyte maturation which may provide insight to the cause of ovarian dysfunction [4]. 
References:

[1] SMB Obayomi et al., Microscopy and Microanalysis 23 (2017), p. 1176.

[2] MG Bianchetti et al., J. Mol. Med. 60 (1982), p. 465.

[3] DP Baluch and DG Capco, Dev. Biol. 317 (2008), p. 46.

[4] We acknowledge the ASU Biology and Society for their generous graduate research award funding.
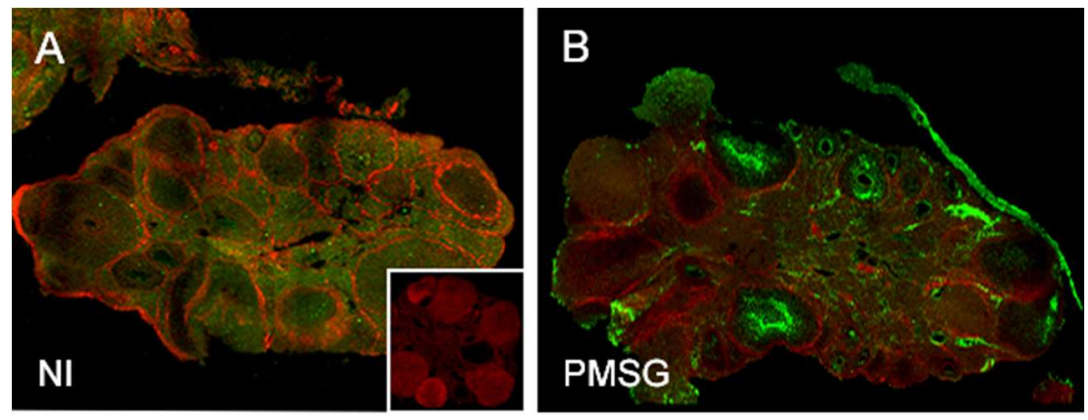

Figure 1. Localization of TAAR1 in Mouse Ovaries. Mouse ovaries are either non-injected (A), or injected with PMSG (B), PMSG and hCG (C) or post treated with a physiological concentration of tyramine (D) after ovary removal. The outline of follicle borders are highlighted with actin
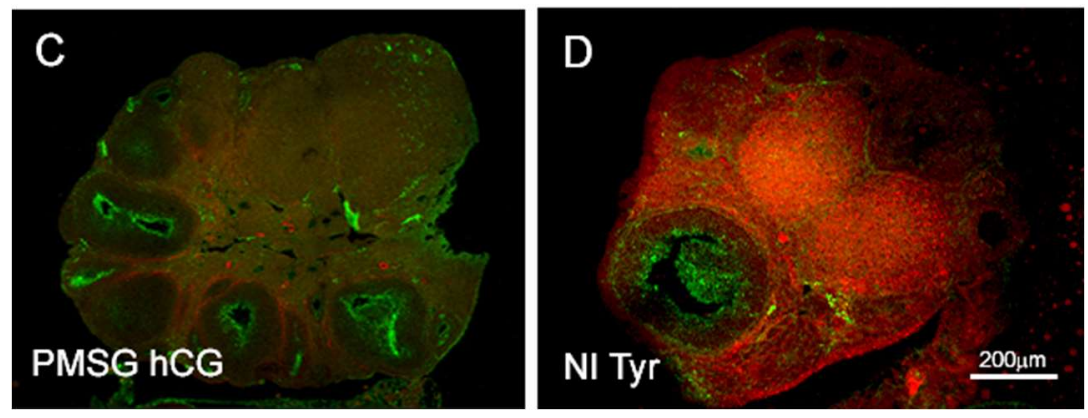
labeled with phalloidin (red). TAAR1 localization is seen in green. Tyramine has been shown to be associated with smooth muscle and is localized at the perimeter of the follicles. It localizes in greater concentration at the corpus luteum possibly to facilitate luteal regression.

Figure 2. Affect of Tyramine on Mouse Ovaries. Image A shows a non-hormonally stimulated ovary with follicles at various levels of maturation with actin/phalloidin labeling in green and DNA/DAPI in gray. Image B shows an ovary after exposure to a physiological concentration of tyramine for 45 minutes. The tyramine-stimulated ovary shows a disrupted organization of the follicles, which is similar in appearance to an ovary that has been super-ovulated. The inset is a histological preparation showing the usual pattern of DNA organization around a primary follicle. Scale bar is $200 / \mathrm{m}$.

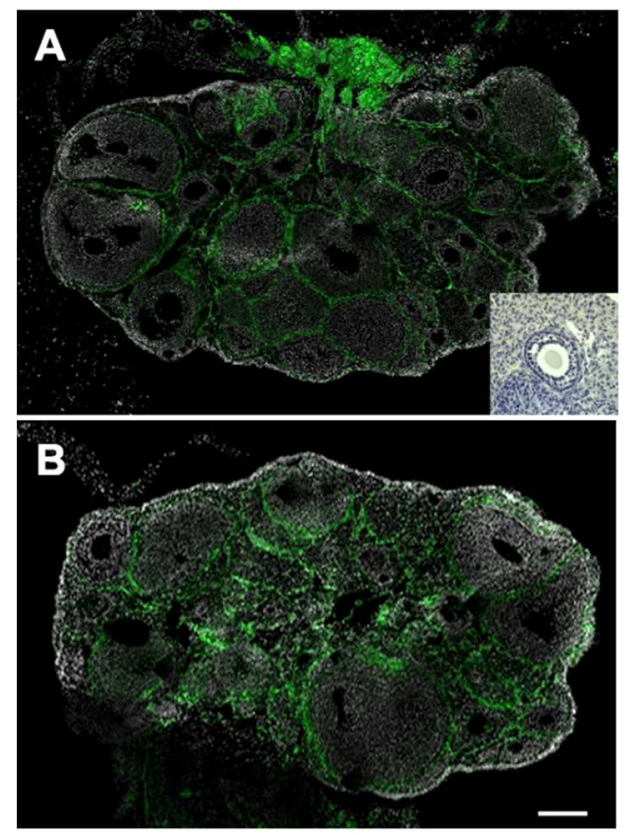

